\title{
A LOWER BOUND IN NEHARI'S THEOREM ON THE POLYDISC
}

\author{
JOAQUIM ORTEGA-CERDÀ AND KRISTIAN SEIP
}

\begin{abstract}
By theorems of Ferguson and Lacey $(d=2)$ and Lacey and Terwilleger $(d>2)$, Nehari's theorem is known to hold on the polydisc $\mathbb{D}^{d}$ for $d>1$, i.e., if $H_{\psi}$ is a bounded Hankel form on $H^{2}\left(\mathbb{D}^{d}\right)$ with analytic symbol $\psi$, then there is a function $\varphi$ in $L^{\infty}\left(\mathbb{T}^{d}\right)$ such that $\psi$ is the Riesz projection of $\varphi$. A method proposed in Helson's last paper is used to show that the constant $C_{d}$ in the estimate $\|\varphi\|_{\infty} \leq C_{d}\left\|H_{\psi}\right\|$ grows at least exponentially with $d$; it follows that there is no analogue of Nehari's theorem on the infinite-dimensional polydisc.
\end{abstract}

This note solves the following problem studied by H. Helson [2, 3]: Is there an analogue of Nehari's theorem on the infinite-dimensional polydisc? By using a method proposed in [3], we show that the answer is negative. The proof is of interest also in the finite-dimensional situation because it gives a nontrivial lower bound for the constant appearing in the norm estimate in Nehari's theorem; we choose to present this bound as our main result.

We first introduce some notation and give a brief account of Nehari's theorem. Let $d$ be a positive integer, $\mathbb{D}$ the open unit disc, and $\mathbb{T}$ the unit circle. We let $H^{2}\left(\mathbb{D}^{d}\right)$ be the Hilbert space of functions analytic in $\mathbb{D}^{d}$ with square-summable Taylor coefficients. Alternatively, we may view $H^{2}\left(\mathbb{D}^{d}\right)$ as a subspace of $L^{2}\left(\mathbb{T}^{d}\right)$ and express the inner product of $H^{2}\left(\mathbb{D}^{d}\right)$ as $\langle f, g\rangle=\int_{\mathbb{T}^{d}} f \bar{g}$, where we integrate with respect to normalized Lebesgue measure on $\mathbb{T}^{d}$. Every function $\psi$ in $H^{2}\left(\mathbb{D}^{d}\right)$ defines a Hankel form $H_{\psi}$ by the relation $H_{\psi}(f g)=\langle f g, \psi\rangle$; this makes sense at least for holomorphic polynomials $f$ and $g$. Nehari's theorem-a classical result [6] when $d=1$ and a remarkable and relatively recent achievement of S. Ferguson and M. Lacey [1] $(d=2)$ and M. Lacey and E. Terwilleger [5] $(d>2)$ in the general case-says that $H_{\psi}$ extends to a bounded form on $H^{2}\left(\mathbb{D}^{d}\right) \times H^{2}\left(\mathbb{D}^{d}\right)$ if and only if $\psi=P_{+} \varphi$ for some bounded function $\varphi$ on $\mathbb{T}^{d}$; here $P_{+}$is the Riesz projection on $\mathbb{T}^{d}$ or, in other words, the orthogonal projection of $L^{2}\left(\mathbb{T}^{d}\right)$ onto $H^{2}\left(\mathbb{D}^{d}\right)$. We define $C_{d}$ as the smallest constant $C$ that can be chosen in the estimate

$$
\|\varphi\|_{\infty} \leq C\left\|H_{\psi}\right\|
$$

where it is assumed that $\varphi$ has minimal $L^{\infty}$ norm. Nehari's original theorem says that $C_{1}=1$.

Theorem. For even integers $d \geq 2$, the constant $C_{d}$ is at least $\left(\pi^{2} / 8\right)^{d / 4}$.

The theorem thus shows that the blow-up of the constants observed in $[4,5]$ is not an artifact resulting from the particular inductive argument used there.

2000 Mathematics Subject Classification. 47B35, 42B30, 32 A35.

The first author is supported by the project MTM2008-05561-C02-01 and and the grant 2009 SGR 1303. The second author is supported by the Research Council of Norway grant 160192/V30. This work was done as part of the research program Complex Analysis and Spectral Problems at Centre de Recerca Matemàtica (CRM), Bellaterra in the spring semester of 2011. The authors are grateful to CRM for its support and hospitality. 
Since clearly $C_{d}$ increases with $d$ and, in particular, we would need that $C_{d} \leq C_{\infty}$ should Nehari's theorem extend to the infinite-dimensional polydisc, our theorem gives a negative solution to Helson's problem.

Nehari's theorem can be rephrased as saying that functions in $H^{1}\left(\mathbb{D}^{d}\right)$ (the subspace of holomorphic functions in $L^{1}\left(\mathbb{T}^{d}\right)$ ) admit weak factorizations, i.e., every $f$ in $H^{1}\left(\mathbb{D}^{d}\right)$ can be written as $f=\sum_{j} g_{j} h_{j}$ with $f_{j}, g_{j}$ in $H^{2}\left(\mathbb{D}^{d}\right)$ and $\sum_{j}\left\|g_{j}\right\|_{2}\left\|h_{j}\right\|_{2} \leq A\|f\|_{1}$ for some constant $A$. Taking the infimum of the latter sum when $g_{j}, h_{j}$ vary over all weak factorizations of $f$, we get an alternate norm (a projective tensor product norm) on $H^{1}\left(\mathbb{D}^{d}\right)$ for which we write $\|f\|_{1, w}$. We let $A_{d}$ denote the smallest constant $A$ allowed in the norm estimate $\|f\|_{1, w} \leq A\|f\|_{1}$. Our proof shows that we also have $A_{d} \geq\left(\pi^{2} / 8\right)^{d / 2}$ when $d$ is an even integer.

Proof of the theorem. We will follow Helson's approach [3] and also use his multiplicative notation. Thus we define a Hankel form on $\mathbb{T}^{\infty}$ as

$$
H_{\psi}(f g)=\sum_{j, k=1}^{\infty} \rho_{j k} a_{j} b_{k}
$$

here $\left(a_{j}\right),\left(b_{j}\right)$, and $\left(\rho_{j}\right)$ are the sequences of coefficients of the power series of the functions $f, g$, and $\psi$, respectively. More precisely, we let $p_{1}, p_{2}, p_{3}, \ldots$ denote the prime numbers; if $j=p_{1}^{\nu_{1}} \cdots p_{k}^{\nu_{k}}$, then $a_{j}$ (respectively $b_{j}$ and $\rho_{j}$ ) is the coefficient of $f$ (respectively of $g$ and $\psi$ ) with respect to the monomial $z_{1}^{\nu_{1}} \cdots z_{k}^{\nu_{k}}$. We will only consider the finite-dimensional case, which means that the coefficients will be nonzero only for indices $j$ of the form $p_{1}^{\nu_{1}} \cdots p_{d}^{\nu_{d}}$. The prime numbers will play no role in the proof except serving as a convenient tool for bookkeeping.

We now assume that $d$ is an even integer and introduce the set

$$
I=\left\{n \in \mathbb{N}: n=\prod_{j=1}^{d / 2} q_{j} \text { and } q_{j}=p_{2 j-1} \text { or } q_{j}=p_{2 j}\right\} .
$$

We define a Hankel form $H_{\psi}$ on $\mathbb{D}^{d}$ by setting $\rho_{n}=1$ if $n$ is in $I$ and $\rho_{n}=0$ otherwise.

We follow [3, pp. 81-82] and use the Schur test to estimate the norm of $H_{\psi}$. It suffices to choose a suitable finite sequence of positive numbers $c_{j}$ with $j$ ranging over those positive integers that divide some number in $I$; for such $j$ we choose

$$
c_{j}=2^{-\Omega(j) / 2},
$$

where $\Omega(j)$ is the number of prime factors in $j$. We then get

$$
\sum_{k} \rho_{j k} c_{k}=2^{d / 2-\Omega(j)} \cdot 2^{-(d / 2-\Omega(j)) / 2}=2^{d / 4} c_{j},
$$

so that $\left\|H_{\psi}\right\| \leq 2^{d / 4}$ by the Schur test.

If $f$ is a function in $H^{1}\left(\mathbb{D}^{d}\right)$ with associated Taylor coefficients $a_{n}$, then

$$
H_{\psi}(f)=\sum_{n} a_{n} \rho_{n} .
$$


We choose

$$
f(z)=\prod_{j=1}^{d / 2}\left(z_{2 j-1}+z_{2 j}\right)
$$

for which $a_{n}=\rho_{n}$ and thus $H_{\psi}(f)=2^{d / 2}$. On the other hand, an explicit computation shows that

$$
\|f\|_{1}=(4 / \pi)^{d / 2}
$$

so that $H_{\psi}$, viewed as a linear functional on $H^{1}\left(\mathbb{D}^{d}\right)$, has norm at least $(\pi / 2)^{d / 2}$. This concludes the proof since it follows that we must have $(\pi / 2)^{d / 2} \leq\|\varphi\|_{\infty}$ and we know from above that $\left\|H_{\psi}\right\| \leq 2^{d / 4}$.

It is worth noting that our application of the Schur test shows that in fact $\left\|H_{\psi}\right\|=2^{d / 4}$ since $\|f\|_{2}=2^{d / 4}$. The fact that $\left|H_{\psi}(f)\right|=\left\|H_{\psi}\right\|\|f\|_{2}$ implies that

$$
\|f\|_{1, w}=\|f\|_{2} \text {. }
$$

In other words, the trivial factorization $f \cdot 1$ is an optimal weak factorization of the function $f$ defined in (1).

\section{REFERENCES}

[1] S. Ferguson and M. Lacey, A characterization of product BMO by commutators, Acta Math. 189 (2002), 143-160.

[2] H. Helson, Dirichlet Series, Henry Helson, 2005.

[3] H. Helson, Hankel forms, Studia Math. 198 (2010), 79-84.

[4] M. Lacey, Lectures on Nehari's theorem on the polydisk, in: Topics in harmonic analysis and ergodic theory, Contemp. Math. 444, Amer. Math. Soc., Providence, RI, 2007, 185-213.

[5] M. Lacey and E. Terwilleger, Hankel operators in several complex variables and product BMO, Houston J. Math. 35 (2009), 159-183.

[6] Z. Nehari, On bounded bilinear forms, Ann. of Math. (2) 65 (1957), 153-162.

Dept. Matemàtica Aplicada i ANÀlisi, Universitat de Barcelona, Gran Via 585, 08071 BarceLONA, SPAIN

E-mail address: jortega@ub.edu

Department of Mathematical Sciences, Norwegian University of SCIEnCE and Technology, NO-7491 TRONDHEIM, NORWAY

E-mail address: seip@math.ntnu.no 